\title{
Trifoliata hybrids rootstocks for 'Lane Late' navel orange in Spain
}

\author{
Pilar Legua ${ }^{1}$, Ricardo Bellver ${ }^{2}$, Juan Bautista Forner ${ }^{3}$, Maria Angeles Forner-Giner ${ }^{3 *}$ \\ ${ }^{1} U M H$ - Plant Science and Microbiology Dept., Ctra Beniel km 3.2 - 03312 - Oribuela, Alicante - Spain.

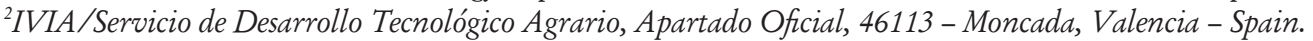 \\ ${ }^{3}$ IVIA/Centro de Citricultura y Producción Vegetal, Apartado Oficial, 46113 - Moncada, Valencia - Spain. \\ *Corresponding author < forner_margin@gva.es> \\ Edited by: Eros Arthur Bohac Francisco
}

\begin{abstract}
Carrizo citrange [Citrus sinensis (L.) Osb. $\times$ Poncirus trifoliata (L.) Raf.] and Cleopatra mandarin (C. reshni Hort. ex Tan.) are the most important rootstocks used in Spain, but they are problematic and it is necessary to search for new rootstocks with better all-round performance. The performance of 'Lane Late' navel orange [Citrus sinensis (L.) Osb] on ten rootstocks was determined in the South of the province of Alicante (Spain). They are Carrizo citrange, Cleopatra mandarin and eight new hybrids obtained at the Instituto Valenciano de Investigaciones Agrarias in Valencia (Spain): 020324 [Troyer citrange (C. sinensis $\times$ P. trifoliata) $\times$ Cleopatra mandarin], Forner-Alcaide 418 (F\&A 418) [Troyer citrange $\times$ common mandarin (C. deliciosa Ten.)], Forner-Alcaide 13 (F\&A 13), 030118, 030127 and 030131 (Cleopatra mandarin $\times$ P. trifoliata) and 030212 and 030230 (Cleopatra mandarin $\times$ Troyer citrange). Soil is clay loam, with $\mathrm{pH} 8.5$ and electric conductivity in the saturation extract at $25^{\circ} \mathrm{C}$ of $5.79 \mathrm{mS} \mathrm{cm}$. Yield was weighed during the first nine harvests, fruit quality was determined in the last three. Pre-harvest fruit-drop was controlled for the $4^{\text {th }}$ until $9^{\text {th }}$ harvests. The trees of 'Lane Late' navel budded on Cleopatra mandarin were the tallest $(2.5 \mathrm{~m})$ and F\&A $418(1.6 \mathrm{~m})$ the shortest of all rootstocks tested. Trees on 030131 hybrid and Carrizo citrange rootstocks had the highest mean yield $(81.2$ and $80.3 \mathrm{~kg}$ per tree per year respectively), while trees on F\&A 418 produced the lowest mean yield (22.3 $\mathrm{kg}$ per tree per year). Trees on 030131, 020324 and 030212 had the highest yield efficiency as total cumulative yield per cubic meter of canopy volume $\left(62.1,58.7\right.$ and $55.9 \mathrm{~kg} \mathrm{~m}^{-3}$ respectively) whereas trees on 030127, F\&A 418 and Cleopatra mandarin had lower yield efficiencies (45.0, 44.4 and $38.6 \mathrm{~kg} \mathrm{~m}^{-3}$, respectively). Pre-harvest fruitdrop was lower in trees grafted on Cleopatra mandarin (24.62\%) and on 030212 (26.61\%), and was also low on F\&A 418 (27.76\%), $020324(28.14 \%)$ and 030230 (29.18\%) rootstocks. Trees on Carrizo citrange and 030127 experienced important fruit-drop ( $40.24 \%$ and $38.27 \%$ respectively). Trees on F\&A 418 had the highest fruit weight and fruit size whereas trees on 030118 induced the lowest ones. The ripeness index was the highest on F\&A 13 (18.3) and lowest on F\&A 418 (15.3), 030212 (15.3).
\end{abstract}

Keywords: citrus rootstock, fruit quality, tree size, yield

\section{Introduction}

'Lane Late' navel orange [Citrus sinensis (L.) Osb] is an important cultivar in Spain that originated as a limb sport of Washington Navel orange in Australia and was first recorded in 1950. It gives rise to vigorous trees and has good productivity with high-quality fruits for fresh consumption. Fruits ripen in December, but are usually harvested in Spain from January to May when other navel oranges have already been harvested (Agustí, 2000).

Carrizo citrange [Citrus sinensis (L.) Osb. $\times$ Poncirus trifoliata (L.) Raf.] is currently the most important rootstock used in Spain, today this represents over $80 \%$ of nursery production. Cleopatra mandarin (C. reshni Hort. ex Tan.) is also used, but represents only $4 \%$ of nursery production (Forner-Giner et al., 2003). Both rootstocks are problematic, and it is necessary to search for new rootstocks with better all-round performance. Many hybrids, some of which are already commercially available, have been obtained by J.B. Forner and co-workers within the citrus rootstock breeding program conducted at the Instituto Valenciano de Investigaciones Agrarias (IVIA), Moncada, Valencia (Spain). The following demonstrate some interesting traits (Table 1) as rootstocks: hybrids of Cleopatra mandarin $\times$ P. trifoliata number 13 (commercially named Forner-Alcaide
13) (Forner et al., 2003; Forner-Giner et al., 2003; Forner-Giner et al., 2009); n 18 (030118), $\mathrm{n}^{\circ} 27$ (030127) and $\mathrm{n}^{\circ} 31$ (030131); Troyer citrange hybrid $(C$. sinensis $\times P$. trifoliata $) \times$ Cleopatra mandarin $\mathrm{n}^{\circ} 24$ (020324); the hybrid of Troyer citrange $\times$ common mandarin (C. deliciosa Ten.) no 18 (sold under the commercial name of Forner Alcaide 418) and the hybrids of Cleopatra mandarin $\times$ Troyer citrange $\mathrm{n}^{\circ} 12(030212)$ and $\mathrm{n}^{\circ}$ 30 (030230).

The objective of the present study was to compare the performance of 'Lane Late' navel orange trees on the most commonly used commercial rootstocks in Spain, plus eight new hybrids, in the citrus-growing areas of the south of Alicante and Murcia provinces in Spain.

\section{Materials and Methods}

Carrizo citrange, Cleopatra mandarin and eight new hybrid selections, obtained in the rootstock breeding program carried out at IVIA since 1974 (Table 1) were tested as rootstocks for 'Lane Late' navel sweet orange. Seeds of Carrizo citrange and Cleopatra mandarin were obtained from the germplasm collection of rootstocks at IVIA and the seeds of the hybrids from the plants obtained in the citrus rootstock breeding program. 
Table 1 - Characteristics of the rootstocks tested for 'Lane Late' orange.

\begin{tabular}{|c|c|c|c|c|c|}
\hline Name & Botanical name & CTV & Calcareous soils & Salinity & Citrus nematode \\
\hline Carrizo citrange & C. sinensis (L.) Osb. $\times$ P. trifoliata (L.) Raf. & Tolerant $^{e}$ & Susceptible $^{e}$ & Susceptible $^{e}$ & Susceptible $e^{e}$ \\
\hline Cleopatra mandarin & C. reshni Hort. ex Tan. & Tolerant $^{\mathrm{e}}$ & Good tolerance $^{\mathrm{e}}$ & Good tolerance $^{e}$ & Susceptible $^{e}$ \\
\hline F\&A 13 & C. reshni $\times P$. trifoliata $\mathrm{n}^{\circ} 13$ & Resistant $^{\mathrm{d}}$ & Susceptible $^{\mathrm{d}}$ & Good tolerance $^{\mathrm{d}}$ & Susceptible $^{f}$ \\
\hline F\&A 418 & $\begin{array}{l}\text { Troyer c. } \times \text { common mandarin }(C . \text { deliciosa } \\
\text { Ten.) } \mathrm{n}^{\circ} 18\end{array}$ & Tolerant $^{\mathrm{b}}$ & Intermediate $^{\mathrm{b}}$ & Intermediate $^{\mathrm{b}}$ & Susceptible ${ }^{\mathrm{b}, \mathrm{f}, \mathrm{g}}$ \\
\hline 020324 & $\begin{array}{l}\text { Troyer citrange }(C \text {. sinensis } \times P \text {. trifoliata }) \times C \text {. } \\
\text { reshni } \mathrm{n}^{\circ} 24\end{array}$ & Tolerant $^{\mathrm{a}}$ & Good tolerance ${ }^{b}$ & Good tolerance $^{\mathrm{b}}$ & Susceptible ${ }^{g}$ \\
\hline 030118 & C. reshni $\times P$. trifoliata $\mathrm{n}^{\circ} 18$ & Resistant $^{\mathrm{b}}$ & Intermediate $^{\mathrm{b}}$ & Good tolerance $^{\mathrm{b}}$ & Resistant $^{\mathrm{f}, \mathrm{g}}$ \\
\hline 030127 & C. reshni $\times P$. trifoliata $\mathrm{n}^{\circ} 27$ & Resistant $^{\mathrm{a}}$ & Susceptible $^{a}$ & Good tolerance ${ }^{\mathrm{a}}$ & Highly resistant ${ }^{g}$ \\
\hline 030131 & C. reshni $\times P$. trifoliata $\mathrm{n}^{\circ} 31$ & Tolerant $^{\mathrm{c}}$ & Tolerant ${ }^{a}$ & Good tolerance ${ }^{a}$ & Highly resistant $^{\mathrm{f}}$ \\
\hline 030212 & C. reshni $\times$ Troyer c. $\mathrm{N}^{\circ} 12$ & Tolerant $^{a}$ & Intermediate $^{a}$ & Good tolerance ${ }^{a}$ & Susceptible ${ }^{g}$ \\
\hline 030230 & C. reshni $\times$ Troyer c. $\mathrm{N}^{\circ} 30$ & Tolerant $^{a}$ & Tolerant $\mathrm{t}^{\mathrm{a}}$ & Intermediate $^{a}$ & Susceptible ${ }^{g}$ \\
\hline
\end{tabular}

anpublished data; ${ }^{\mathrm{b}}$ Forner et al., 1996; ${ }^{\mathrm{c}}$ Forner et al., 2000; ${ }^{\mathrm{d}}$ Forner et al., 2003; ${ }^{\mathrm{e}}$ Newcomb, 1978; ${ }^{\mathrm{f}}$ Verdejo-Lucas et al., 1997; ${ }^{\mathrm{g}}$ Verdejo-Lucas et al., 2000 .

All nursery processes were performed in an aphid-proof greenhouse, equipped with a cooling system, at temperatures ranging between $18^{\circ} \mathrm{C}$ and $27^{\circ} \mathrm{C}$ and relative humidity of about $80 \%$. On May $28^{\text {th }} 1996$, one year after budding, the nursery trees were planted in a randomized block design with 14 singletree replications for each scion-stock combination in Elche, a village near Alicante, Spain (38 $\left.14^{\prime} \mathrm{N}, 0^{\circ} 41^{\prime} \mathrm{W}\right)$. Tree spacing was $4.5 \times 4 \mathrm{~m}$. The block was surrounded by guard rows on all four sides. Elche is located near the Mediterranean Sea (about $10 \mathrm{~km}$ ). It has clay loam soil, with $\mathrm{pH}$ of $8.5, \mathrm{CaCO}_{3} 44.4 \%$, active calcium carbonate $17.1 \%$ and electric conductivity in the saturation extract at $25{ }^{\circ} \mathrm{C}$ of $5.79 \mathrm{~m} \mathrm{~S} \mathrm{~cm}^{-1}$.

Standard cultural practices for this variety were used with drip irrigation and chemical weed-control. Water $\mathrm{pH}$ was 8.0 while electrical conductivity was $0.8 \mathrm{~m} \mathrm{~S} \mathrm{~cm}^{-1}$ up to 2000 . From 2001 water $\mathrm{pH}$ was 7.8 with electrical conductivity ranging from 2.0 to $3.5 \mathrm{~m} \mathrm{~S} \mathrm{~cm}^{-1}$ and 400 to $500 \mathrm{mg} \mathrm{kg}^{-1}$ of B. Fertilizer was applied beginning in the $2^{\text {nd }}$ year and increased annually. After the $6^{\text {th }}$ year, the amounts applied were: ammonium nitrate $(33 \% \mathrm{~N}) 2 \mathrm{~kg}$ per tree, monoammonium phosphate $0.5 \mathrm{~kg}$ per tree, $\mathrm{KNO}_{3} 0.7 \mathrm{~kg}$ per tree and iron chelate $10 \mathrm{~g}$ per tree in springtime. After three years of cultivation, trees were hand-pruned annually after harvest, as is usual in Spain.

In January 2007, after being planted in the field for 11 years, tree height and canopy diameter were measured for all the trees. Canopy volume was calculated using Turrell's formula (1946). In April or May each tree was harvested and the recently fallen fruits were collected. Yield was measured for nine years $\left(1998 / 99\right.$ until 2006/2007). From the $4^{\text {th }}$ harvest, the number of fruits harvested from each tree was also counted and the mean weight per fruit calculated. To evaluate differences between rootstocks in terms of pre-harvest drop, no treatments with 2,4-DP (2-ethylhexyl ester or dichlorprop-p) were applied and on the $4^{\text {th }}$ (May 10), $5^{\text {th }}$ (May 5), $6^{\text {th }}$ (April 29), $7^{\text {th }}$ (May 12), $8^{\text {th }}$ (May 25) and $9^{\text {th }}$ (May 23) harvests, the fruits that dropped from November until harvest were collected and weighed fortnightly. Fallen fruit weights were added to harvested fruit weights.
To estimate the first year of full production, for each rootstock, a statistical comparison was made with the means of yields of $3^{\text {rd }}$ to $9^{\text {th }}$, considering the full production to be first year displaying no statistical difference with some of the means of the posterior harvests. Cumulative yield for 1998/99 through 2006/07 (nine-year cumulative yield) was used to calculate the yield efficiency as the ratio of cumulative yield to canopy volume. The alternate bearing index (ABI) was calculated by dividing the difference between two consecutive harvests by the sum of two yields $\times 100 \%$ for the nine harvests. If the index exceeds $50 \%$, this means that the tree is in alternate bearing, while the tree is in regular bearing if this index is below $50 \%$.

Fruit quality was determined for the $5^{\text {th }}, 6^{\text {th }}$ and $9^{\text {th }}$ harvests. After the harvest, 25-fruit samples were collected from the harvest containers of each tree and fruit diameter, rind thickness, juice content, total soluble solids (TSS) and total acids (TA) were determined (Forner-Giner et al., 2003). Ripening index was determined as the TSS/TA ratio. Peel-color index was not determined because the fruits were fully colored on harvesting.

Experimental data were analyzed using Statgraphic Plus. For the percentage of pre-harvest fruit-drop and ABI, the data were arcsin transformed prior to analysis. Statistical analyses were performed using SPSS 18.0 for Windows. A basic descriptive statistical analysis was followed by an analysis on variance test for mean comparisons. The method used to discriminate among the means (Multiple Range Test) was the Fisher's Least Significant Difference (LSD) procedure $(p<$ $0.05)$.

\section{Results and Discussion}

Eleven years after field planting, the trees on Cleopatra mandarin were the biggest (tree height $2.5 \mathrm{~m}$, canopy diameter $3.7 \mathrm{~m}$, and canopy volume $18.0 \mathrm{~m}^{3}$ ) (Table 2). The smallest trees were on F\&A 418 (tree high $1.6 \mathrm{~m}$, canopy diameter $2.3 \mathrm{~m}$, and canopy volume $\left.4.5 \mathrm{~m}^{3}\right)$. Trees on Carrizo citrange $\left(14.0 \mathrm{~m}^{3}\right)$, $030127\left(13.4 \mathrm{~m}^{3}\right)$, and $030230\left(12.7 \mathrm{~m}^{3}\right)$ did not differ in canopy 
volume. The F\&A $13\left(11.0 \mathrm{~m}^{3}\right), 020324\left(10.7 \mathrm{~m}^{3}\right), 030212$ $\left(10.3 \mathrm{~m}^{3}\right)$ and $030118\left(9.2 \mathrm{~m}^{3}\right)$ also did not differ among each other. Trees on $030131\left(11.9 \mathrm{~m}^{3}\right)$ were not different from those grafted on 020324, F\&A 13, 030127, 030212 and 030230. Trees on Carrizo citrange, Cleopatra mandarin, 020324, 030131 and 030230 did not present Fe deficiency and were on excellent green color from the time they were planted. Trees on F\&A 418, F\&A 13, 030118, 030127 and 030212 exhibited some Fe deficiency despite the application of iron chelate in springtime.

All rootstocks yielded their first fruits in 1998, two years after planting (Table 3). Carrizo citrange, 020324, 030118, 030131, 030212 and 030230 yield increased until the $5^{\text {th }}$ year (means of the $4^{\text {th }}$ harvest with no differences with $8^{\text {th }}$ and with $9^{\text {th }}$ for Carrizo and 030131). Yield of trees on F\&A 418

Table 2 - Tree size of Lane Late navel orange on ten rootstocks 11 years after planting ${ }^{2}$.

\begin{tabular}{|c|c|c|c|}
\hline Rootstock & Tree height & Canopy diameter & Canopy volume \\
\hline & ----------- & - $\mathrm{m}$---------- & $\mathrm{m}^{3}$ \\
\hline Carrizo citrange & $2.4 \mathrm{~b}$ & $3.4 \mathrm{~b}$ & $14.0 \mathrm{~b}$ \\
\hline $\begin{array}{l}\text { Cleopatra } \\
\text { mandarin }\end{array}$ & $2.5 \mathrm{a}$ & $3.7 \mathrm{a}$ & $18.0 \mathrm{a}$ \\
\hline F\&A 13 & $2.1 \mathrm{de}$ & $3.2 \mathrm{bc}$ & $11.0 \mathrm{def}$ \\
\hline F\&A 418 & $1.6 \mathrm{f}$ & $2.3 \mathrm{e}$ & $4.5 \mathrm{~g}$ \\
\hline 020324 & $2.2 \mathrm{bcd}$ & $3.0 \mathrm{~cd}$ & $10.7 \mathrm{ef}$ \\
\hline 030118 & $2.0 \mathrm{e}$ & $3.0 \mathrm{~d}$ & $9.2 \mathrm{f}$ \\
\hline 030127 & $2.3 \mathrm{bc}$ & $3.3 \mathrm{~b}$ & $13.4 \mathrm{bc}$ \\
\hline 030131 & $2.2 \mathrm{~cd}$ & $3.2 \mathrm{~b}$ & $11.9 \mathrm{cde}$ \\
\hline 030212 & $2.2 \mathrm{bcd}$ & $3.0 \mathrm{~d}$ & $10.3 \mathrm{ef}$ \\
\hline 030230 & $2.3 \mathrm{bc}$ & $3.2 \mathrm{bc}$ & $12.7 \mathrm{bcd}$ \\
\hline
\end{tabular}

${ }^{a}$ Mean separation within columns (LSD test, $p<0.05$ ). increased until $4^{\text {th }}$ year (means of $3^{\text {rd }}$ and $4^{\text {th }}$ harvests not different) and Cleopatra mandarin, F\&A 13, and 030127 until $6^{\text {th }}$ year $\left(5^{\text {th }}\right.$ harvest) which may be considered the first years of full production. It would appear that the F\&A 418 bear fruit earlier.

Trees on hybrid 030131 (81.2 kg per tree per year) and on Carrizo citrange (80.3 kg per tree per year) had the highest mean yield, followed by Cleopatra mandarin $(76.1 \mathrm{~kg}$ per tree per year). Trees on hybrids 030127 (66.1 kg per tree per year), 020324 (66.0 kg per tree per year), 030230 (64.1 kg per tree per year), 030212 (64.0 kg per tree per year) and F\&A 13 (63.5 kg per tree per year) formed a third group with high mean yield presenting differences with the other rootstocks. Trees on 030118 gave low mean yield $(52.7 \mathrm{~kg}$ per tree per year) whereas those grafted on F\&A 418 rootstock the lowest (22.3 kg per tree per year), with statistical differences compared to the other rootstocks studied (Table 3). Trees on 030131 gave the highest yield efficiency $\left(62.1 \mathrm{~kg} \mathrm{~m}^{-3}\right)$ but did not differ from trees grafted onto $020324\left(58.7 \mathrm{~kg} \mathrm{~m}^{-3}\right)$ and $030212\left(55.9 \mathrm{~kg} \mathrm{~m}^{-3}\right)$. The lowest yield efficient trees were those grafted onto Cleopatra mandarin $\left(38.6 \mathrm{~kg} \mathrm{~m}^{-3}\right)$, F\&A $418\left(44.4 \mathrm{~kg} \mathrm{~m}^{-3}\right)$ and $030127\left(45.0 \mathrm{~kg} \mathrm{~m}^{-3}\right)$, with no differences between them. Trees on F\&A $13\left(52.9 \mathrm{~kg} \mathrm{~m}^{-3}\right), 030118$ $\left(52.7 \mathrm{~kg} \mathrm{~m}^{-3}\right)$, Carrizo citrange $\left(52.6 \mathrm{~kg} \mathrm{~m}^{-3}\right)$ and $030230(47.0$ $\mathrm{kg} \mathrm{m}^{-3}$ ) gave an intermediate yield efficiency, without differences between them.

The alternate bearing index (ABI) differed among some of the rootstocks, although none of them exhibited alternate bearing index. 030131 (20\%), Carrizo citrange (21\%) and $020324(24 \%)$ were the rootstocks with most uniform productivity. Pre-harvest fruit-drop was important (Table 4). The average for the last six harvests $\left(4^{\text {th }}\right.$ till $9^{\text {th }}$ year $)$, trees on Carrizo citrange (40.24\%) and on 030127 (38.27\%) suffered the highest percentage of fruit-drop, followed by trees on F\&A 13 (36.84\%). Trees on Cleopatra mandarin

Table 3 - Yield and yield efficiency of 'Lane Late' navel orange trees on ten rootstocks planted in May 1996.

\begin{tabular}{|c|c|c|c|c|c|c|c|c|c|c|c|c|}
\hline \multirow{3}{*}{ Rootstooks } & \multicolumn{9}{|c|}{ Yield (kg per tree) } & \multirow{3}{*}{ Means $^{a}$} & \multirow{3}{*}{$\begin{array}{l}\text { Yield }^{\mathrm{a}, \mathrm{b}} \\
\text { efficiency }\end{array}$} & \multirow{3}{*}{$\mathrm{ABI}^{\mathrm{a}}$} \\
\hline & $1^{\text {st }}$ & $2^{\text {nd }}$ & $3^{\text {rd }}$ & $4^{\text {th }}$ & $5^{\text {th }}$ & $6^{\text {th }}$ & $7^{\text {th }}$ & $8^{\text {th }}$ & $9^{\text {th }}$ & & & \\
\hline & $1998 / 99$ & $1999 / 00$ & $2000 / 01$ & $2001 / 02$ & $2002 / 03$ & $2003 / 04$ & $2004 / 05$ & $2005 / 06$ & $2006 / 07$ & & & \\
\hline & & & & & & & & & & $\begin{array}{l}\mathrm{kg} \text { per tree } \\
\text { per year }\end{array}$ & $\mathrm{kg} \mathrm{m}^{-3}$ & $\%$ \\
\hline Carrizo citrange $^{c}$ & 8.1 & 38.0 & $49.5 \mathrm{~d}$ & $87.4 \mathrm{c}$ & $112.4 \mathrm{~b}$ & $111.1 \mathrm{~b}$ & $133.1 \mathrm{a}$ & $85.9 \mathrm{c}$ & $97.1 \mathrm{c}$ & $80.3 \mathrm{a}$ & $52.6 \mathrm{bcd}$ & $21 \mathrm{e}$ \\
\hline Cleopatra mandarin $^{c}$ & 3.1 & 22.7 & $35.8 \mathrm{e}$ & $66.1 \mathrm{~d}$ & $98.8 \mathrm{c}$ & $99.6 \mathrm{c}$ & $142.3 \mathrm{a}$ & $91.4 \mathrm{c}$ & $125.1 \mathrm{~b}$ & $76.1 \mathrm{~b}$ & $38.6 \mathrm{f}$ & $27 \mathrm{bcd}$ \\
\hline $\mathrm{F} \& \mathrm{~A} 13^{\mathrm{c}}$ & 1.6 & 9.7 & 33.8 e & $60.6 \mathrm{~d}$ & $94.3 \mathrm{~b}$ & $88.5 \mathrm{~b}$ & $121.8 \mathrm{a}$ & $75.3 \mathrm{c}$ & 85.9 bc & $63.5 \mathrm{c}$ & $52.9 \mathrm{bc}$ & $30 \mathrm{ab}$ \\
\hline $\mathrm{F} \& \mathrm{~A} 418^{\mathrm{c}}$ & 1.4 & 1.9 & $9.9 \mathrm{e}$ & $17.8 \mathrm{de}$ & $36.0 \mathrm{~b}$ & $25.4 \mathrm{~cd}$ & $30.6 \mathrm{bc}$ & $32.2 \mathrm{bc}$ & $45.5 \mathrm{a}$ & $22.3 \mathrm{e}$ & $44.4 \mathrm{ef}$ & $32 \mathrm{a}$ \\
\hline $020324^{c}$ & 6.5 & 27.7 & $39.3 \mathrm{~d}$ & $68.1 \mathrm{c}$ & $89.3 \mathrm{~b}$ & $84.3 \mathrm{~b}$ & $118.9 \mathrm{a}$ & $67.0 \mathrm{c}$ & $93.1 \mathrm{~b}$ & $66.0 \mathrm{c}$ & $58.7 \mathrm{ab}$ & $24 \mathrm{de}$ \\
\hline $030118^{c}$ & 1.2 & 8.2 & $26.0 \mathrm{~d}$ & $51.2 \mathrm{c}$ & $73.2 \mathrm{~b}$ & $68.2 \mathrm{~b}$ & $101.3 \mathrm{a}$ & $65.9 \mathrm{bc}$ & $79.2 \mathrm{~b}$ & $52.7 \mathrm{~d}$ & $52.7 \mathrm{bcd}$ & $31 \mathrm{ab}$ \\
\hline $030127^{c}$ & 1.9 & 9.2 & $29.9 \mathrm{~d}$ & $55.1 \mathrm{c}$ & $95.1 \mathrm{~b}$ & $89.3 \mathrm{~b}$ & $132.8 \mathrm{a}$ & $85.0 \mathrm{~b}$ & $101.9 \mathrm{~b}$ & $66.1 \mathrm{c}$ & 45.0 def & $30 \mathrm{abc}$ \\
\hline $030131^{c}$ & 9.4 & 35.8 & $57.8 \mathrm{~d}$ & $83.3 \mathrm{c}$ & $107.7 \mathrm{~b}$ & $117.8 \mathrm{~b}$ & $140.2 \mathrm{a}$ & $92.2 \mathrm{c}$ & $86.7 \mathrm{c}$ & $81.2 \mathrm{a}$ & $62.1 \mathrm{a}$ & $20 \mathrm{e}$ \\
\hline $030212^{c}$ & 7.3 & 21.8 & $30.7 \mathrm{~d}$ & $58.3 \mathrm{c}$ & $98.9 \mathrm{ab}$ & $86.6 \mathrm{~b}$ & $110.9 \mathrm{a}$ & $69.4 \mathrm{c}$ & $92.0 \mathrm{~b}$ & $64.0 \mathrm{c}$ & $55.9 \mathrm{ab}$ & $26 \mathrm{~cd}$ \\
\hline $030230^{c}$ & 4.8 & 25.2 & $34.0 \mathrm{~d}$ & $65.1 \mathrm{c}$ & $97.7 \mathrm{ab}$ & $90.3 \mathrm{~b}$ & $106.3 \mathrm{a}$ & $54.3 \mathrm{c}$ & $98.9 \mathrm{ab}$ & $64.1 \mathrm{c}$ & $47.0 \mathrm{cde}$ & $27 \mathrm{bcd}$ \\
\hline
\end{tabular}

${ }^{\mathrm{a}}$ Mean separation within columns by LSD test $(p<0.05)$; ${ }^{\mathrm{b}}$ As total cumulated yields per cubic meter of canopy volume; ${ }^{\mathrm{c}}$ Mean separation within rows from $3^{\text {rd }}$ to $9^{\text {th }}$ harvests by LSD test $(p<0.05)$. 
Table 4 - Pre-harvest fruit-drop of Lane Late navel orange trees on ten rootstocks in \% over total yield.

\begin{tabular}{lccccccc}
\hline \multirow{2}{*}{ Rootstocks } & \multicolumn{7}{c}{ Harvests } \\
\cline { 2 - 8 } & $4^{\text {th }}$ & $5^{\text {th }}$ & $6^{\text {th }}$ & $7^{\text {th }}$ & $8^{\text {th }}$ & $9^{\text {th }}$ & Means $^{\text {a }}$ \\
\hline Carrizo citrange & 46.13 & 53.12 & 26.75 & 28.69 & 25.18 & 61.60 & $40.24 \mathrm{a}$ \\
$\begin{array}{l}\text { Cleopatra } \\
\text { mandarin }\end{array}$ & 25.68 & 37.54 & 14.20 & 12.10 & 20.89 & 37.29 & $24.62 \mathrm{f}$ \\
F\&A 13 & 46.63 & 43.10 & 23.39 & 16.98 & 34.71 & 56.23 & $36.84 \mathrm{~b}$ \\
\hline F\&A 418 & 24.15 & 36.31 & 24.02 & 15.06 & 24.89 & 42.15 & $27.76 \mathrm{e}$ \\
020324 & 30.61 & 35.60 & 15.87 & 14.45 & 25.91 & 46.39 & $28.14 \mathrm{e}$ \\
\hline 030118 & 38.17 & 45.03 & 18.43 & 13.52 & 33.60 & 46.35 & $32.52 \mathrm{~cd}$ \\
030127 & 49.80 & 43.56 & 32.69 & 20.11 & 28.46 & 55.00 & $38.27 \mathrm{ab}$ \\
\hline 030131 & 42.01 & 39.32 & 21.75 & 14.58 & 23.84 & 59.45 & $33.49 \mathrm{c}$ \\
030212 & 30.65 & 27.66 & 19.96 & 11.71 & 23.28 & 46.41 & $26.61 \mathrm{ef}$ \\
030230 & 30.92 & 37.76 & 15.67 & 16.46 & 31.96 & 42.31 & $29.18 \mathrm{de}$ \\
\hline
\end{tabular}

${ }^{a}$ Mean separation within column by LSD test $(p<0.05)$.

Table 5 - Weight, diameter and peel thickness of fruits of 'Lane Late' navel orange on ten rootstocks.

\begin{tabular}{|c|c|c|c|c|c|c|c|c|c|c|c|c|}
\hline \multirow{3}{*}{ Rootstocks } & \multirow{2}{*}{\multicolumn{3}{|c|}{$\begin{array}{c}\text { Fruit weight }(\mathrm{g}) \\
\text { Harvests }\end{array}$}} & \multirow{3}{*}{ Means ${ }^{\mathrm{a}}$} & \multirow{2}{*}{\multicolumn{3}{|c|}{$\begin{array}{c}\text { Fruit diameter }(\mathrm{mm}) \\
\text { Harvests }\end{array}$}} & \multirow{3}{*}{ Means $^{\mathrm{a}}$} & \multirow{2}{*}{\multicolumn{3}{|c|}{$\begin{array}{c}\text { Peel thickness }(\mathrm{mm}) \\
\text { Harvests }\end{array}$}} & \multirow{3}{*}{ Means $^{\mathrm{a}}$} \\
\hline & & & & & & & & & & & & \\
\hline & $7^{\text {th }}$ & $8^{\text {th }}$ & $9^{\text {th }}$ & & $7^{\text {th }}$ & $8^{\text {th }}$ & $9^{\text {th }}$ & & $7^{\text {th }}$ & $8^{\text {th }}$ & $9^{\text {th }}$ & \\
\hline Carrizo citrange & 219.4 & 189.1 & 176.8 & $229.2 \mathrm{~d}$ & 76.9 & 72.8 & 66.3 & $72.0 \mathrm{c}$ & 3.9 & 4.2 & 4.2 & $4.1 \mathrm{c}$ \\
\hline $\begin{array}{l}\text { Cleopatra } \\
\text { mandarin }\end{array}$ & 224.6 & 227.2 & 181.4 & $229.4 \mathrm{~d}$ & 80.0 & 76.7 & 66.9 & $74.5 \mathrm{~b}$ & 4.0 & 4.0 & 4.3 & $4.1 \mathrm{c}$ \\
\hline F\&A 13 & 232.8 & 241.1 & 186.2 & $234.9 \mathrm{~cd}$ & 77.0 & 72.6 & 69.4 & $73.0 \mathrm{c}$ & 4.0 & 3.6 & 4.5 & $4.1 \mathrm{c}$ \\
\hline F\&A 418 & 244.9 & 222.2 & 171.5 & $262.1 \mathrm{a}$ & 79.4 & 76.2 & 69.9 & $75.2 \mathrm{a}$ & 4.1 & 5.0 & 5.4 & $4.8 \mathrm{a}$ \\
\hline 020324 & 214.5 & 219.8 & 171.8 & $231.9 \mathrm{~d}$ & 80.5 & 75.2 & 65.8 & $73.8 \mathrm{~b}$ & 4.3 & 4.3 & 4.5 & $4.4 \mathrm{~b}$ \\
\hline 030118 & 200.3 & 223.9 & 170.9 & 213.9 e & 76.3 & 75.0 & 64.9 & $72.1 \mathrm{c}$ & 3.9 & 3.8 & 3.9 & $3.9 \mathrm{~d}$ \\
\hline 030127 & 241.4 & 231.5 & 169.0 & $226.7 \mathrm{~d}$ & 78.4 & 73.5 & 66.4 & $72.8 \mathrm{c}$ & 4.0 & 3.9 & 4.0 & $4.0 \mathrm{~cd}$ \\
\hline 030131 & 243.8 & 228.9 & 191.8 & 246.4 b & 79.2 & 78.0 & 66.5 & $74.6 \mathrm{~b}$ & 4.2 & 4.3 & 4.6 & $4.4 \mathrm{~b}$ \\
\hline 030212 & 215.2 & 205.9 & 184.3 & $226.3 \mathrm{~d}$ & 76.3 & 70.6 & 65.8 & $70.9 \mathrm{~d}$ & 4.2 & 3.6 & 4.6 & $4.1 \mathrm{c}$ \\
\hline 030230 & 225.4 & 224.9 & 174.8 & $242.7 \mathrm{bc}$ & 80.1 & 74.1 & 67.6 & $73.9 \mathrm{~b}$ & 4.1 & 4.3 & 4.5 & $4.3 \mathrm{~b}$ \\
\hline
\end{tabular}

${ }^{a}$ Mean separation within columns by LSD test $(p<0.05)$.

(24.62\%) were the least affected by pre-harvest fruit-drop, without difference with the trees on 030212 (26.61\%). Also little affected were the trees on F\&A 418 (27.76 \%), 020324 $(28.14 \%)$ and $030230(29.18 \%)$ that did not differ between them and with 030212. Trees on 030118 and on 030131 lost a third of their yield before harvest (32.52\% and $33.49 \%$ respectively). As most fruits are destined for fresh consumption, fruit size, juice content and TSS/TA ratio are of great importance in Spain. Rootstock affected all fruit quality variables studied.

The highest mean of fruit weight for the $7^{\text {th }}$ till $9^{\text {th }}$ harvest was obtained on F\&A 418 hybrid rootstock (262.1 g per fruit) (Table 5). The hybrid 030131 (246.4 g per fruit) also induced good fruit weight, but did not differ from 030230 (242.7 g per fruit) and F\&A 13 (234.9 g per fruit). There were no differences between the latter rootstock and 020324
(231.9 g per fruit), Cleopatra mandarin (229.4 g per fruit) Carrizo citrange (229.2 g per fruit), 030127 (226.7 g per fruit) or 030212 (226.3 g per fruit).

Trees on F\&A 418 gave the largest fruit diameter (75.2 $\mathrm{mm})$, followed by 030131 (74.6 mm), Cleopatra mandarin $(74.5 \mathrm{~mm}), 030230(73.9 \mathrm{~mm})$ and $020324(73.8 \mathrm{~mm})$, which also differed from the other rootstocks but without differences between them. Another group was formed by F\&A 13 (73.2 mm), 030127 (72.8 mm), $030118(72.1 \mathrm{~mm})$ and Carrizo citrange $(72.0 \mathrm{~mm})$. Trees on 030212 had the smallest fruits (70.9 mm).

Fruits produced by trees on F\&A 418 had the thickest peel $(4.8 \mathrm{~mm})$ (Table 5). The 030131 (4.4 mm), 020324 (4.4 $\mathrm{mm})$ and $030230(4.3 \mathrm{~mm})$ differed from the other rootstocks but not from each other. Cleopatra mandarin, F\&A 13, Carrizo citrange, 030212 (all four rootstocks with $4.1 \mathrm{~mm}$ of 
Table 6 - Fruit quality of 'Lane Late' navel orange on ten rootstocks: internal variables.

\begin{tabular}{|c|c|c|c|c|c|c|c|c|c|c|c|c|c|c|c|c|}
\hline \multirow[t]{3}{*}{ Rootstocks } & \multicolumn{4}{|c|}{ Juice $(\%)$} & \multicolumn{4}{|c|}{ Total soluble solids ( $\%)$} & \multicolumn{4}{|c|}{ Total acids (\%) } & \multicolumn{4}{|c|}{$\begin{array}{l}\text { Total soluble solids } \\
\text { Total acids }^{-1}\end{array}$} \\
\hline & \multicolumn{3}{|c|}{ Harvests } & \multirow{2}{*}{ - Means ${ }^{\mathrm{a}}$} & \multicolumn{3}{|c|}{ Harvests } & \multirow{2}{*}{ Means $^{a}$} & \multicolumn{3}{|c|}{ Harvests } & \multirow{2}{*}{ Means $^{a}$} & \multicolumn{3}{|c|}{ Harvests } & \multirow{2}{*}{ Means $^{\mathrm{a}}$} \\
\hline & $7^{\text {th }}$ & $8^{\text {th }}$ & $9^{\text {th }}$ & & $7^{\text {th }}$ & $8^{\text {th }}$ & $9^{\text {th }}$ & & $7^{\text {th }}$ & $8^{\text {th }}$ & $9^{\text {th }}$ & & $7^{\text {th }}$ & $8^{\text {th }}$ & $9^{\text {th }}$ & \\
\hline Carrizo citrange & 56.7 & 54.6 & 50.4 & $53.9 \mathrm{ab}$ & 14.5 & 15.4 & 14.7 & $14.9 \mathrm{a}$ & 0.92 & 0.93 & 0.10 & $0.95 \mathrm{a}$ & 15.7 & 16.5 & 14.7 & $15.6 \mathrm{~cd}$ \\
\hline Cleopatra mandarin & 55.3 & 56.5 & 48.1 & $53.3 \mathrm{~b}$ & 12.9 & 14.8 & 15.2 & $14.3 \mathrm{~b}$ & 0.73 & 0.84 & 0.99 & $0.85 \mathrm{~b}$ & 17.6 & 17.5 & 15.4 & $16.8 \mathrm{~b}$ \\
\hline F\&A 13 & 55.9 & 57.2 & 49.7 & $54.3 \mathrm{ab}$ & 13.1 & 15.4 & 14.8 & $14.4 \mathrm{~b}$ & 0.69 & 0.77 & 0.92 & $0.79 \mathrm{c}$ & 18.9 & 20.1 & 16.1 & $18.3 \mathrm{a}$ \\
\hline F\&A 418 & 56.3 & 53.9 & 46.7 & $52.3 \mathrm{c}$ & 12.9 & 15.4 & 15.3 & $14.5 \mathrm{ab}$ & 0.90 & 0.92 & 0.10 & $0.95 \mathrm{a}$ & 14.3 & 16.7 & 14.9 & $15.3 \mathrm{~d}$ \\
\hline 020324 & 56.0 & 55.9 & 47.6 & $53.2 \mathrm{~b}$ & 13.4 & 14.8 & 15.3 & $14.5 \mathrm{ab}$ & 0.82 & 0.96 & 0.97 & $0.92 \mathrm{ab}$ & 16.3 & 15.4 & 15.7 & $15.8 \mathrm{c}$ \\
\hline 030118 & 58.7 & 57.4 & 51.5 & $55.9 \mathrm{a}$ & 13.8 & 14.8 & 15.3 & $14.6 \mathrm{ab}$ & 0.87 & 0.79 & 0.11 & $0.91 \mathrm{ab}$ & 15.8 & 18.7 & 14.2 & $16.2 \mathrm{c}$ \\
\hline 030127 & 57.3 & 58.1 & 50.8 & $55.4 \mathrm{a}$ & 13.3 & 14.8 & 15.1 & $14.4 \mathrm{~b}$ & 0.78 & 0.87 & 0.11 & $0.92 \mathrm{ab}$ & 17.0 & 17.0 & 13.6 & $15.9 \mathrm{c}$ \\
\hline 030131 & 56.4 & 56.3 & 48.8 & $53.8 \mathrm{ab}$ & 13.1 & 15.3 & 15.6 & $14.7 \mathrm{ab}$ & 0.73 & 0.81 & 0.11 & $0.87 \mathrm{~b}$ & 17.9 & 19.0 & 14.3 & $17.1 \mathrm{~b}$ \\
\hline 030212 & 56.0 & 54.9 & 50.3 & $53.7 \mathrm{ab}$ & 13.8 & 15.5 & 15.7 & $15.0 \mathrm{a}$ & 0.88 & 0.10 & 0.10 & $0.98 \mathrm{a}$ & 15.6 & 15.3 & 15.0 & $15.3 \mathrm{~d}$ \\
\hline 030230 & 56.9 & 53.7 & 45.4 & $52.0 \mathrm{c}$ & 14.4 & 15.1 & 15.1 & $14.9 \mathrm{a}$ & 0.88 & 0.84 & 0.87 & $0.87 \mathrm{~b}$ & 16.3 & 17.9 & 17.3 & $17.2 \mathrm{~b}$ \\
\hline
\end{tabular}

${ }^{a}$ Mean separation within columns by LSD test $(p<0.05)$.

peel thickness) and $030127(4.0 \mathrm{~mm})$ did not differ from each other. The thinnest peel corresponded to fruits of trees on 030118 (3.9 mm).

There were small differences between rootstocks with respect to internal fruit quality variables (Table 6). All means for juice content exceeded $52 \%$. Fruits from trees on 030118 and 030127 had the highest juice content. F\&A 13, Carrizo citrange, 030131 and 030212 did not differ from the latter two and with Cleopatra mandarin and 020324 . Fruits produced by trees grafted on F\&A 418 and 030230 had the lowest mean juice content and were different from the other rootstocks. Total soluble solids (TSS) ranged from $14.3 \%$ to $15.0 \%$. Fruits from trees on Carrizo citrange, 030212 and 030230 had the highest TSS values while Cleopatra mandarin, F\&A 13 and 030127 had the lowest one. The other rootstocks: 030118 , 030131020324 and F\&A 418 were not different from the first and the second groups. The highest total acid (TA) content was found in fruits from trees on Carrizo citrange $(0.95 \%)$, F\&A $418(0.95 \%)$ and $030212(0.98 \%)$ while the lowest was found on fruits of F\&A 13 (0.79\%). Ripeness index (TSS $\mathrm{TA}^{-1}$ ) was highest on F\&A 13 (18.3) and lowest on F\&A 418 (15.3), 030212 (15.3) and Carrizo citrange (15.6).

The hybrid 030131 performed well with 'Lane Late' Navel orange scions. It had the highest cumulative yield and the best yield efficiency, higher than Carrizo citrange. The fruit quality was good, with excellent fruit weight and size, juice content over $50 \%$ and mean total soluble solids (TSS) of $14.7 \%$ for three harvests. In previous trials this hybrid of Cleopatra mandarin $\times P$ trifoliata (number 31 ) proved tolerant to citrus tristeza virus, with good tolerance to calcareous soils and high tolerance to salinity (unpublished data) and high resistance to the citrus nematode Tylenchulus semipenetrans Cobb (Verdejo-Lucas et al., 1997 and 2000). The hybrid of Troyer citrange $\times$ Cleopatra mandarin number 24 (020324) also performed well in this trial, having a good yield efficiency. In previous trials (unpublished data) it was tolerant to citrus tristeza virus, and showed good tolerance to calcareous soils and salinity.
However, it is susceptible to citrus nematode (Verdejo-Lucas et al., 1997 and 2000).

Cleopatra mandarin, 030127 and F\&A 418 had the lowest yield efficiency. Six out of the eight new hybrid rootstocks performed better than Cleopatra mandarin in this important trait.

The yield efficiency of trees on Carrizo citrange was not different from 020324, 030212, F\&A 13, 030118, 030230 and 030127 rootstocks. Bassal (2009) on studying 'Marisol' Clementine, reported that Cleopatra mandarin was the least productive rootstock. F\&A 13 is resistant to citrus tristeza virus (CTV), susceptible to lime-induced chlorosis (González-Mas et al., 2009; Llosá et al., 2009), and resistant to salinity (FornerGiner et al., 2009). The yield efficiency is similar to Carrizo citrange, but higher than Cleopatra mandarin (Table 3), but tree size and canopy volume are smaller than Carrizo citrange, as previously described (Forner-Giner et al., 2003).

Pre-harvest fruit-drop is currently an important problem in Spain and to reduce this problem, the trees are sprayed with 2,4-DP (2-ethylhexyl ester or dichlorprop-p). In addition, the excess of Spanish production of some cultivars lengthens the harvest period and an important part of the crop is lost. For these reasons it is an excellent trait for a rootstock to retain the ripened fruit. In this experiment, Cleopatra mandarin and the new hybrids 030230, 020324, F\&A 418 and 030212 had the lowest pre-harvest fruit-drop. In other trials (unpublished data) F\&A 418 retained almost all of the ripened fruits for several months (less than 1C fruit drop). Carrizo citrange, 030127 and F\&A 13 were the rootstocks with highest pre-harvest fruitdrop. Trees on 030131 and 030118 rootstocks dropped fruit intermediately. F\&A 13 and F\&A 418 have been registered for protection in the European Union by the Instituto Valenciano de Investigaciones Agrarias (IVIA) and the Instituto Nacional de Investigación y Tecnología Agraria y Alimentaria (INIA) (Forner et al., 2003; Forner-Giner et al., 2003 and FornerGiner et al., 2009). Similar protection is being sought for the hybrids 030131 and 020324 , by the IVIA. 


\section{Acknowledgements}

This work was financed by the Instituto Nacional de Investigación y Tecnología Agraria y Alimentaria (INIA), grants $\mathrm{n}^{\circ}$ SC94-037-C2, SC98-103-C2, RTA02-104 and RTA2005-00136, RTA2008-00060, and the Instituto Valenciano de Investigaciones Agrarias (IVIA).

\section{References}

Agustí, M. 2000. Citriculture. Mundi Prensa Press, Madrid, Spain. (in Spanish).

Bassal, M.A. 2009. Growth, yield and fruit quality of 'Marisol' clementine grown on tour rootstocks in Egypt. Scientia Horticulturae 119: 132137.

Forner, J.B.; Forner-Giner, M.A.; Alcaide, A. 2003. Forner-Alcaide 5 and Forner-Alcaide 13: Two new citrus rootstocks released in Spain. HortScience 38: 629-630.

Forner-Giner, M.A.; Alcaide, A.; Primo-Millo, E.; Forner, J.B. 2003. Performance of 'Navelina' orange on 14 rootstocks in Northern Valencia (Spain). Scientia Horticulturae 98: 223-232.
Forner-Giner, M.A.; Primo-Millo, E.; Forner, J.B. 2009. Performance of Forner-Alcaide 5 and Forner-Alcaide 13, hybrids of Cleopatra mandarin $\times$ Poncirus trifoliata, as salinity-tolerant citrus rootstocks. Journal of the American Pomological Society 63: 72-80.

González-Mas, M.C.; Llosá M.J.; Quijano A.; Forner-Giner, M.A. 2009. Rootstock effects on leaf photosynthesis in Navelina trees grown in calcareous soils. Hortscience 44: 280-283.

Llosá, M.J., Bermejo, A., Cano, A., Quiñones, A., Forner-Giner M.A. 2009. The citrus rootstocks Cleopatra mandarin, Poncirus trifoliata, FornerAlcaide 5 and Forner-Alcaide 13 vary in susceptibility to iron deficiency chlorosis. Journal of the American Pomological Society 63: 160-167.

Turrell, F.M. 1946. Tables of Surfaces and Volumes of Spheres and of Prolate and Oblate Spheroids and Spheroidal Coefficients. University of California Press, Berkeley, CA, USA.

Verdejo-Lucas, S.; Sorribas, F.J; Forner J.B., Alcaide, A. 1997. Screening hybrid citrus rootstocks for resistance to Tylenchulus semipenetrans Cobb. HortScience 32: 1116-1119.

Verdejo-Lucas, S.; Sorribas, FJ.; Forner, J.B.; Alcaide, A. 2000. Resistance of hybrid citrus rootstocks to a mediterranean biotype of Tylenchulus semipenetrans Cobb. HortScience 35: 269-273.

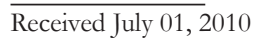

Accepted January 19, 2011 